\title{
Signatures of Chiral Magnetic Effect in the Collisions of Isobars
}

\author{
Shuzhe Shi, ${ }^{1}$ Hui Zhang, ${ }^{2,3}$ Defu Hou, ${ }^{2},{ }^{*}$ and Jinfeng Liao ${ }^{4, \dagger}$ \\ ${ }^{1}$ Department of Physics, McGill University, 3600 University Street, Montreal, QC, H3A 2T8, Canada. \\ ${ }^{2}$ Institute of Particle Physics (IOPP) and Key Laboratory of Quark and Lepton Physics (MOE), \\ Central China Normal University, Wuhan 430079, China. \\ ${ }^{3}$ Guangdong Provincial Key Laboratory of Nuclear Science, Institute of Quantum Matter, \\ South China Normal University, Guangzhou 510006, China. \\ ${ }^{4}$ Physics Department and Center for Exploration of Energy and Matter, \\ Indiana University, $2401 \mathrm{~N}$ Milo B. Sampson Lane, Bloomington, IN 47408, USA.
}

(Dated: January 29, 2021)

\begin{abstract}
Quantum anomaly is a fundamental feature of chiral fermions. In chiral materials the microscopic anomaly leads to nontrivial macroscopic transport processes such as the Chiral Magnetic Effect (CME), which has been in the spotlight lately across disciplines of physics. The quark-gluon plasma (QGP) created in relativistic nuclear collisions provides the unique example of a chiral material consisting of intrinsically relativistic chiral fermions. Potential discovery of CME in QGP is of utmost significance, with extensive experimental searches carried out over the past decade. A decisive new collider experiment, dedicated to detecting CME in the collisions of isobars, was performed in 2018 with analysis now underway. In this paper, we develop the state-of-the-art theoretical tool for describing CME phenomenon in these collisions and propose an appropriate isobar subtraction strategy for best background removal. Based on that, we make quantitative predictions for signatures of CME in the collisions of isobars. A new and robust observable that is independent of axial charge uncertainty - the ratio between isobar-subtracted $\gamma-$ and $\delta$ - correlators, is found to be $-(0.41 \pm 0.27)$ for event-plane measurement and $-(0.90 \pm 0.45)$ for reaction-plane measurement.
\end{abstract}

PACS numbers: 25.75.-q, 25.75.Gz, 25.75.Ld

Introduction. - The investigation of novel quantum transport in chiral materials is a rapidly growing area of research that has attracted significant interests and activities recently from a broad range of physics disciplines such as high energy physics, condensed matter physics, astrophysics, cold atomic gases, etc. Chiral materials are many-body quantum systems that consist of massless fermions (i.e. chiral fermions) that are either fundamental particles or emergent quasi-particles behaving as chiral fermions. A notable example of the former, is the so-called quark-gluon plasma (QGP) which is a new phase of hadronic matter existing at primordially high temperatures available in the early Universe and which is now recreated in laboratories by high energy nuclear collisions [1-6]. The novel examples of the latter include the latest discovered topological phases of condensed matter systems known as Dirac and Weyl semimetals [7-10].

The most salient feature of chiral fermions is the chiral anomaly under the presence of gauge interactions. Chiral materials manifest such microscopic quantum peculiarity through unique macroscopic anomalous transport processes, which are forbidden in normal environment yet become possible (and necessary) in such chiral materials. A famous example is the so-called Chiral Magnetic Effect (CME), predicting the generation of an electric current in chiral materials as response to an applied magnetic field. The CME is a remarkable example as a new kind of quantum electricity that one may call "magnetricity". The observation of CME in various physical systems is of fundamental importance. In semimetal systems the
CME-induced transport has been measured via observables like negative magnetoresistance [11-14]. In the subatomic chiral material, i.e. the quark-gluon plasma created in relativistic nuclear collisions, enthusiastic efforts have been made to look for its evidence at Relativistic Heavy Ion Collider (RHIC) and Large Hadron Collider (LHC) [15-23]. Despite many measurements accumulated so far from RHIC and LHC with encouraging hints, the interpretation of these data remains inconclusive due to background contamination. The main challenge is that the flow-driven background contribution is proportional to elliptic flow and mimicking the desired CME signal, as clearly revealed by event-shape analysis in e.g. [21, 23]. See discussions in e.g. [1, 6, 24, 25].

An unambiguous observation of CME in the subatomic system would be its first confirmation in a chiral material of intrinsic relativistic fermions. Its detection would also provide tantalizing experimental verification for the high-temperature restoration of a spontaneously broken global symmetry (the chiral symmetry) which is a fundamental prediction of the Quantum Chromodynamics (QCD). It would additionally open a unique window for characterizing the intriguing topological fluctuations of gluon fields - the non-Abelian gauge fields of QCD. Given such importance, a decisive isobaric collision experiment has been carried out in 2018 at RHIC, with the dedicated goal of discovering the CME [26-28]. The basic idea, as initially suggested by Voloshin in [26], is to contrast the CME-sensitive observables in two different colliding systems, the RuRu and the $\mathrm{ZrZr}$, where the $\mathrm{Ru}$ 
and $\mathrm{Zr}$ are a pair of isobar nuclei with the same nucleon numbers $(A=96)$ but different nuclear charges $(Z=44$ and $Z=40$ respectively). The expectation is that the two systems will have the same backgrounds while noticeably different CME signals due to the difference in their nuclear charge and thus magnetic field strength. This experiment offers the unique opportunity to detect CME in such collisions and the data analysis is actively underway. A precise characterization of the signals and backgrounds is critically needed.

The present work focuses on making theoretical predictions for the signatures of CME in the isobar experiment. For that purpose, we develop a state-of-the-art tool and the first of its kind, the EBE-AVFD (event-byevent anomalous-viscous fluid dynamics), that can characterize CME signals from dynamical anomalous transport as well as account for background correlations in a realistic heavy ion collision environment. With this powerful tool, we compute CME observables and report key results that shall provide unique insights into forthcoming experimental measurements.

Methodology. - In heavy ion collisions, the Chiral Magnetic Effect induces an electric current along the magnetic field, approximately perpendicular to the reaction plane (RP) [29]. Therefore a CME-induced charge separation across the reaction plane is expected and can be measured by the charge asymmetry in azimuthal correlations of same-sign (SS) and opposite-sign (OS) charged hadron pairs. There are however non-CME background correlations contributing substantially to relevant observables, with resonance decays and local charge conservation being dominant sources. To unambiguously extract the CME signal has proven extremely challenging. To resolve such pressing issue and pave the way for potential discovery of CME would require: (1) a sophisticated and realistic simulation framework that can quantitatively characterize backgrounds and predict the CME signatures; (2) a model-independent analysis approach to subtract out backgrounds. The methodology we adopt in this study aims precisely to address these, by developing the EBE-AVFD framework and by a suitable isobar comparison strategy.

To quantitatively describe CME-induced signatures in the collisions, one needs to compute the anomalous charge transport current in dynamically evolving bulk fluid modeled by relativistic viscous fluid dynamics $[30,31]$. (We note that there are efforts in simulating CME based on non-hydrodynamic models [32-34].) Based on theoretical foundation from [35], we present here a full-fledged fluid dynamical realization of CME transport in modeling heavy ion collisions. This new framework is built upon our earlier Anomalous-Viscous Fluid Dynamics (AVFD) [36, 37], which describes the dynamical evolution of fermion currents (i.e. the quark currents of various flavors and chirality) perturbatively on top of the neutral bulk fluid. A few crucial elements how- ever were missing, including background correlation implementations, event-by-event fluctuations and a hadron cascade stage after hydro-stage.

In the present work, we've successfully addressed these challenges by developing the Event-By-Event Anomalous-Viscous Fluid Dynamics (EBE-AVFD). In the EBE-AVFD, the initial state fluctuations are fully accounted for by event-wise sampling for bulk entropy density and fermion axial charge density. Following the end of hydrodynamic stage (using VISHNU hydro simulations) for each bulk event evolution, hadrons are sampled by maintaining charge conservations and then further evolved through hadron cascade stage via URQMD simulations. This framework for the first time allows a quantitative and consistent evaluation of both CME signals and background correlations within the same realistic bulk evolution. The EBE-AVFD represents state-ofthe-art tool for reliable predictions of CME signatures in heavy ion collisions.

The key to the success of the isobaric contrast idea, is to make sure that one has two collections of RuRu and $\mathrm{ZrZr}$ collision events that must be identical in their bulk properties (multiplicity and elliptic flow $v_{2}$ ). In conventional analysis one would select events based on centrality (i.e. multiplicity) and then compare RuRu with $\mathrm{ZrZr}$ systems at the same centrality. Recent simulations of initial geometry in these collisions however suggest difference at a few percent level in their elliptic eccentricity (for the same centrality) due to uncertainty in the nucleon distributions of the isobar nuclei [38-40]. This presents enough of concern which may complicate the supposedly "clean" isobar comparison, given the small CME signal. To ensure a successful isobar contrast, we propose a new strategy for comparing the isobaric systems, namely to use a joint (multiplicity + elliptic flow) event selection method [39]. We have extensively verified the effectiveness of this strategy based on joint (multiplicity + elliptic flow) event selection for a variety of nucleon distributions, both with event-by-event simulations of initial conditions and with the final state events from EBE-AVFD simulations. More specifically, the elliptic flow coefficient $v_{2}$ in our simulations is computed by first identifying event plane $\Psi_{E P}$ with all charged particles within the rapidity range $y \in(1.5,4.0)$ and then evaluating $v_{2}=\left\langle\cos \left(2 \phi-2 \Psi_{E P}\right)\right\rangle$ for charged particles at mid-rapidity $y \in(-1,1)$. We find that between the isobar pairs, the relative difference in geometry is reduced to the level of $\sim 0.1 \%$ while the relative difference in magnetic field remains at the $10 \sim 25 \%$ level. Therefore, such an isobar comparison and subtraction strategy allows an unambiguous contrast between $\mathrm{RuRu}$ and $\mathrm{ZrZr}$ to reveal potential magnetic-field-driven CME signal despite uncertainty about the initial nucleon distributions.

Predictions. - Here we present predictions for isobaric collisions from our EBE-AVFD simulations. Focusing on events corresponding to $40 \sim 50 \%$ centrality range, we 
have generated ten millions of collision events for each of the RuRu and ZrZr systems. For each system, EBEAVFD simulations are done for $10^{5}$ different hydrodynamic initial profiles, with 100 hadron cascade events following each hydrodynamic profile. We treat each hadroncascade outcome independently and use all these $10^{7}$ events together for our analysis of final state observables such as multiplicity, elliptic flow and charge-dependent correlations. We apply the identical joint cut for multiplicity $N^{c h}$ and elliptic flow $v_{2}: 65 \leq N_{|y|<1}^{c h} \leq 96$ and $0.05<v_{2}<0.25$ for both systems. We have done a consistency check by comparing the post-selection events from both RuRu and ZrZr which are found to be identical, with $\left\langle N_{c h}\right\rangle=80.4$ (standard deviation 8.5) and $\left\langle v_{2}\right\rangle=0.1132$ (standard deviation 0.046 ). This procedure thus guarantees the same bulk medium and background correlations for the isobar pairs.

With the selected events we perform analysis of CMEmotivated observables, focusing on the absolute difference between RuRu and ZrZr systems which would subtract out the background portion. What potentially remains from such subtraction would be the pure CME signal. This allows revealing characteristic features expected for a pure CME signal (obtained only after removal of backgrounds via isobar subtraction). The strength of such a pure signal shall be quadratically dependent on the initial axial charge. The correlations from such a signal shall be along the magnetic field direction which would have different degrees of correlation with the event-plane (EP) and the reaction-plane (RP) [29, 4150]. These features would provide key validation of the EBE-AVFD predictions for isobaric collisions.

The CME current leads to a charge separation along the magnetic field direction that can be measured with azimuthal correlations such as the $\gamma$-correlator [51]:

$$
\gamma^{\alpha \beta}=\left\langle\cos \left(\phi^{\alpha}+\phi^{\beta}-2 \Psi_{2}\right)\right\rangle
$$

where $\Psi_{2}$ should ideally be the reaction plane (RP) but is practically identified via the event plane (EP). The correlation of pairs with same electric charge, $\gamma^{S S}$, has $\{\alpha \beta\} \rightarrow\{++\}$ or $\{--\}$ while that for opposite charged pairs, $\gamma^{O S}$, has $\{\alpha \beta\} \rightarrow\{+-\}$ or $\{-+\}$. To maximize the signal and reduce the backgrounds, one can further examine the difference between the correlation of same and opposite charged pairs, $\gamma^{O S-S S}=\gamma^{O S}-\gamma^{S S}$. Another closely related observable is the $\delta$-correlator $[24,52]$

$$
\delta^{\alpha \beta}=\left\langle\cos \left(\phi^{\alpha}-\phi^{\beta}\right)\right\rangle \text {. }
$$

with $\delta^{O S-S S}=\delta^{O S}-\delta^{S S}$.

The CME would contribute to the above correlators as $: \gamma_{C M E}^{O S-S S} \rightarrow 2\left\langle a_{1}^{2} \cos \left(2 \Psi_{B}-2 \Psi_{2}\right)\right\rangle$ and $\delta_{C M E}^{O S-S S} \rightarrow$ $-2\left\langle a_{1}^{2}\right\rangle$ where $a_{1}$ is the event-wise charge separation dipole along the magnetic field direction $\Psi_{B}$ with $\cos \left(2 \Psi_{B}-2 \Psi_{2}\right)$ capturing the azimuthal de-correlation between $\Psi_{B}$ and $\Psi_{2}$. However, CME contributions could not be easily extracted from current measurements of $\gamma$ and $\delta$ correlators due to dominant non-CME backgrounds [52-59]. This is where the isobar contrast would be uniquely valuable. We will focus on their difference: $\quad \gamma_{R u-Z r}^{O S-S S}=\gamma_{R u R u}^{O S-S S}-\gamma_{Z r Z r}^{O S-S S}$ and $\delta_{R u-Z r}^{O S-S S}=$ $\delta_{R u R u}^{O S-S S}-\delta_{Z r Z r}^{O S-S S}$.

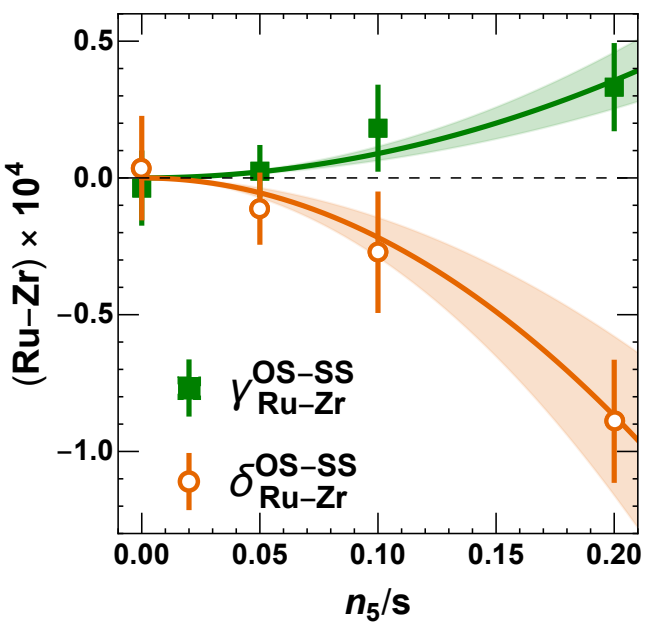

FIG. 1. (color online) EBE-AVFD predictions for $\gamma_{R u-Z r}^{O S-S S}$ (solid square) and $\delta_{R u-Z r}^{O S-S S}$ (open circle) with respect to eventplane (EP) (i.e. $\Psi_{2} \rightarrow \Psi_{E P}$ ) for $n_{5} / s=0 \%, 5 \%, 10 \%$ and $20 \%$ respectively. Vertical error bars are statistical uncertainty from simulations. The curves are quadratic fitting results with shaded uncertainty bands.

In Fig. 1 we show EBE-AVFD predictions for $\gamma_{R u-Z r}^{O S-S S}$ and $\delta_{R u-Z r}^{O S-S S}$ with respect to event-plane (EP) geometry (i.e. $\Psi_{2} \rightarrow \Psi_{E P}$ ), for four different levels of initial axial charge density (normalized by initial bulk entropy density $s$ ) at $n_{5} / s=0 \%, 5 \%, 10 \%$ and $20 \%$ respectively. The quantitative consistency between $\gamma$ and $\delta$ correlators would provide sufficient validation of CME-signal and help constrain the uncertainty in initial axial charge.

The CME-induced correlations should depend quadratically on the $n_{5}$. Simulation results indeed show such a trend, with quadratic fitting curves presented in Fig. 1 and summarized below:

$$
\begin{gathered}
\left.\gamma_{R u-Z r}^{O S-S S}\right|_{E P} \simeq(0.89 \pm 0.51) \times 10^{-3} \times\left(\frac{n_{5}}{s}\right)^{2} \\
\left.\delta_{R u-Z r}^{O S-S S}\right|_{E P} \simeq-(2.17 \pm 0.72) \times 10^{-3} \times\left(\frac{n_{5}}{s}\right)^{2}
\end{gathered}
$$

We further propose a new observable $\zeta_{\text {isobar }}$ built from the ratio between the two correlators:

$$
\zeta_{\text {isobar }}^{E P} \equiv \frac{\left.\gamma_{R u-Z r}^{O S-S S}\right|_{E P}}{\left.\delta_{R u-Z r}^{O S-S S}\right|_{E P}} \simeq-(0.41 \pm 0.27)
$$

This new ratio is independent of the (uncertain) initial axial charge and therefore provides a robust test of CME. The ratio essentially reflects the azimuthal de-correlation 
$\cos \left(2 \Psi_{B}-2 \Psi_{E P}\right)$, which is independently computed to be about 0.46 and quantitatively consistent with the above ratio. These features are specific to pure CME signal and are manifested only after isobar subtraction.

Comparison of measurements with respect to reaction plane (RP) and to event plane (EP) could help decipher CME signal [56, 60], as the magnetic field has different degrees of azimuthal de-correlations with RP and with EP. Experimentally one may use the spectator plane (from e.g. ZDC) as a proxy for RP. It would be interesting to examine correlators with respect to $R P$.

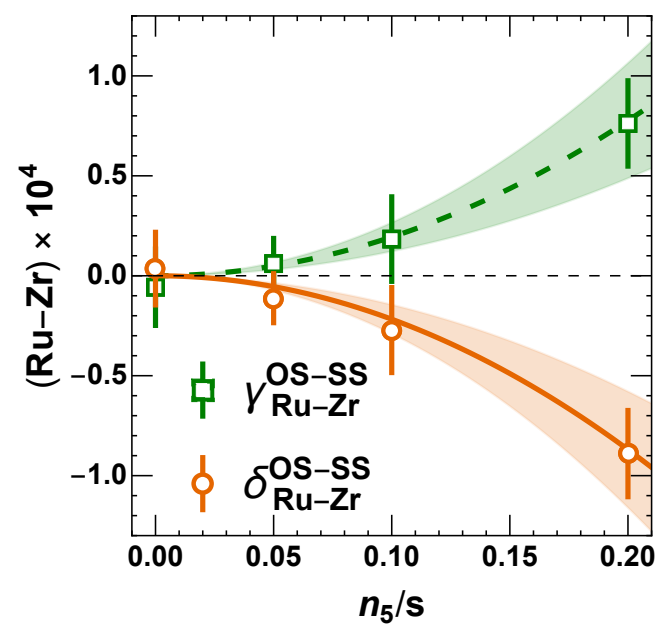

FIG. 2. (color online) Same as Fig. 1 but for correlations measured with respect to the reaction-plane (RP) (i.e. $\Psi_{2} \rightarrow$ $\left.\Psi_{R P}\right)$.

In Fig. 2 we show EBE-AVFD predictions for $\gamma_{R u-Z r}^{O S-S S}$ and $\delta_{R u-Z r}^{O S-S S}$ with respect to reaction-plane (i.e. $\Psi_{2} \rightarrow$ $\left.\Psi_{R P}\right)$. Compared with EP results in Fig. 1, $\gamma$ correlator becomes larger due to a stronger correlation between magnetic field and the RP, as uniquely expected for pure CME signal. Fig. 2 also presents quadratic fitting curves:

$$
\begin{gathered}
\left.\gamma_{R u-Z r}^{O S-S S}\right|_{R P} \simeq(1.94 \pm 0.72) \times 10^{-3} \times\left(\frac{n_{5}}{s}\right)^{2} \\
\left.\delta_{R u-Z r}^{O S-S S}\right|_{R P} \simeq-(2.17 \pm 0.72) \times 10^{-3} \times\left(\frac{n_{5}}{s}\right)^{2}
\end{gathered}
$$

The $\mathrm{RP}$ result for the ratio observable $\zeta_{\text {isobar }}$ is:

$$
\zeta_{\text {isobar }}^{R P} \equiv \frac{\left.\begin{array}{c}
\gamma_{R u-Z r}^{O S-S S} \\
\delta_{R P}^{O S-S S}
\end{array}\right|_{R P}}{\delta_{R u-Z r}} \simeq-(0.90 \pm 0.45)
$$

This RP ratio is about twice that from EP, in quantitative consistency with the expected de-correlation factor $\cos \left(2 \Psi_{B}-2 \Psi_{R P}\right)$ of about 0.95 . The EP and RP measurements of these correlators and the proposed ratios would together provide a stringent test for validating the CME signatures.

Event-shape analysis provides a way of revealing the backgrounds, showing the dependence of $\gamma$-correlator on bulk $v_{2}[21,23]$. A pure CME signal, on the other hand, should be (nearly) independent of event shape. This provides an important consistency check for CME signal from isobar subtraction. In Fig. 3 we show EBEAVFD results for $\gamma_{R u-Z r}^{O S-S S}$ and $\delta_{R u-Z r}^{O S-S S}$ versus event shape in three bins: $v_{2} \in(0.01,0.055), v_{2} \in(0.055,0.11)$ and $v_{2} \in(0.11,0.30)$. We indeed observe that isobarsubtracted $\gamma$ and $\delta$ are independent of event shape.

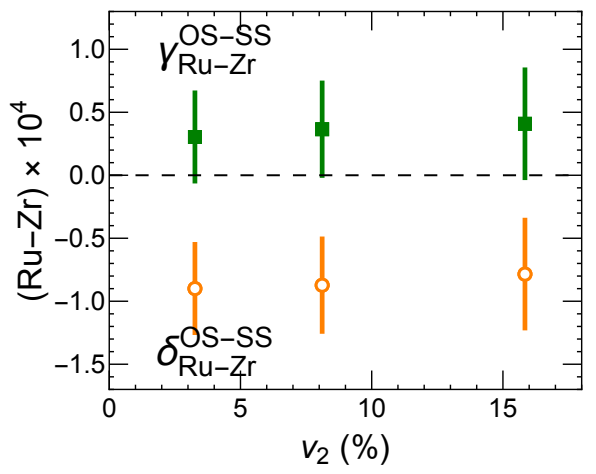

FIG. 3. (color online) EBE-AVFD predictions for observables $\gamma_{R u-Z r}^{O S-S S}$ and $\delta_{R u-Z r}^{O S-S S}$ as a function of bin-wise elliptic flow $v_{2}$ from event-shape analysis with three identical bins for $\mathrm{RuRu}$ and $\mathrm{ZrZr}$ systems. The simulation results are obtained with $n_{5} / s=20 \%$.

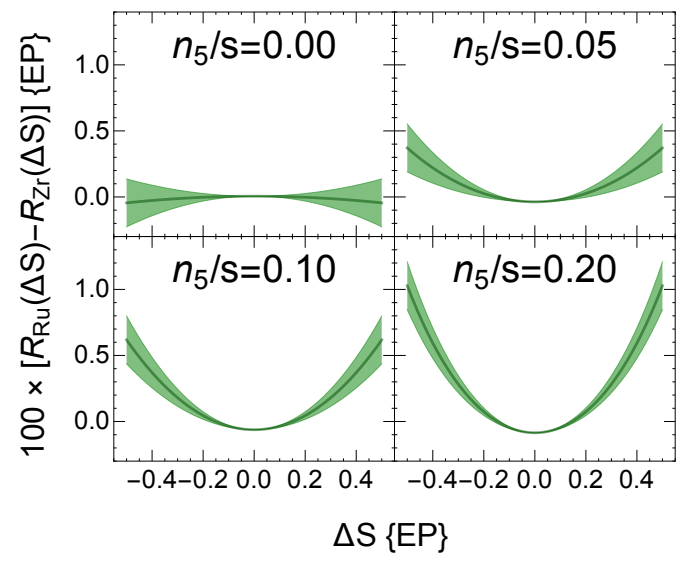

FIG. 4. (color online) EBE-AVFD predictions for the Rcorrelator distributions for $n_{5} / s=0 \%, 5 \%, 10 \%$ and $20 \%$ respectively.

A number of other CME-sensitive correlators have also been proposed [61-63]. As an example, the socalled $R$-correlator (-see [61, 62] for detailed definition and discussions) has demonstrated a certain sensitivity to the presence of CME. In Fig. 4 we show the EBEAVFD results for the the isobar-subtracted R-correlator, $\left[R_{R u}(\Delta S)-R_{Z r}(\Delta S)\right]$ for $n_{5} / s=0 \%, 5 \%, 10 \%$ and $20 \%$ respectively. It appears flat for the none-CME case $\left(n_{5} / s=0 \%\right)$ while becomes more and more upward concave with increasing CME signal. Measurement of Rcorrelator with enough statistics should provide further validation of the CME signal. 
Conclusion. - In this work we've presented a comprehensive set of quantitative predictions for the signatures of Chiral Magnetic Effect (CME) in the isobaric collisions at RHIC. Reliable predictions for key observables of such a decisive high-profile experiment is critically needed and becomes possible only with the development of the EBEAVFD framework reported in the present study. This novel tool has allowed us to characterize a number of unique features of CME signals in these collisions and to propose the best strategy for an unambiguous validation of CME with a multitude of measurements. These results represent the state-of-the-art understanding of the problem, offers valuable insights for the ongoing data analysis and interpretation, and would help significantly advance the current search of CME in collider experiments.

Given the predicted signal strength and the currently projected measurement precision, we conclude optimistically about the likelihood for a successful extraction of CME signatures in the collisions of isobars. If confirmed indeed, such a detection would not only be the first observation of CME in a subatomic material with intrinsically relativistic chiral fermions, but also provide the tantalizing evidence of QCD chiral symmetry restoration in the quark-gluon plasma as well as the unique manifestation of the elusive QCD gluon topological fluctuations. Establishing the CME phenomenon in the subatomic chiral matter would also have far-reaching implications for the study of CME in other areas of research such as condensed matter physics.

\section{ACKNOWLEDGMENTS}

This work is supported in part by the NSFC Grants No. 11735007 and No. 11875178, by the NSF Grant No. PHY-1913729 and by the U.S. Department of Energy, Office of Science, Office of Nuclear Physics, within the framework of the Beam Energy Scan Theory (BEST) Topical Collaboration. SS is grateful to the Natural Sciences and Engineering Research Council of Canada for support. The computation of this research was performed on IU's Big Red II cluster, that is supported by Lilly Endowment, Inc., through its support for the Indiana University Pervasive Technology Institute, and by the Indiana METACyt Initiative which was also partly supported by Lilly Endowment, Inc.

*houdf@mail.ccnu.edu.cn

† liaoji@indiana.edu

[1] D. E. Kharzeev, J. Liao, S. A. Voloshin, and G. Wang, "Chiral magnetic and vortical effects in high-energy nuclear collisions-A status report," Prog. Part. Nucl. Phys. 88, 1-28 (2016), arXiv:1511.04050 [hep-ph].
[2] Vladimir A. Miransky and Igor A. Shovkovy, "Quantum field theory in a magnetic field: From quantum chromodynamics to graphene and Dirac semimetals," Phys. Rept. 576, 1-209 (2015), arXiv:1503.00732 [hep-ph].

[3] Kenji Fukushima, "Extreme matter in electromagnetic fields and rotation," Prog. Part. Nucl. Phys. 107, 167199 (2019), arXiv:1812.08886 [hep-ph].

[4] Jinfeng Liao, "Anomalous transport effects and possible environmental symmetry 'violation' in heavy-ion collisions," Pramana 84, 901-926 (2015), arXiv:1401.2500 [hep-ph].

[5] Koichi Hattori and Xu-Guang Huang, "Novel quantum phenomena induced by strong magnetic fields in heavy-ion collisions," Nucl. Sci. Tech. 28, 26 (2017), arXiv:1609.00747 [nucl-th].

[6] Adam Bzdak, Shinichi Esumi, Volker Koch, Jinfeng Liao, Mikhail Stephanov, and $\mathrm{Nu} \mathrm{Xu}$, "Mapping the Phases of Quantum Chromodynamics with Beam Energy Scan," Phys. Rept. 853, 1-87 (2020), arXiv:1906.00936 [nuclth].

[7] N. P. Armitage, E. J. Mele, and Ashvin Vishwanath, "Weyl and Dirac Semimetals in Three Dimensional Solids," Rev. Mod. Phys. 90, 015001 (2018), arXiv:1705.01111 [cond-mat.str-el].

[8] Binghai Yan and Claudia Felser, "Topological Materials: Weyl Semimetals," Ann. Rev. Condensed Matter Phys. 8, 337-354 (2017), arXiv:1611.04182 [cond-mat.mtrl-sci].

[9] M. Zahid Hasan, Su-Yang Xu, Ilya Belopolski, and ShinMing Huang, "Discovery of Weyl fermion semimetals and topological Fermi arc states," Ann. Rev. Condensed Matter Phys. 8, 289-309 (2017), arXiv:1702.07310 [condmat.mtrl-sci].

[10] A. A. Burkov, "Weyl Metals," Ann. Rev. Condensed Matter Phys. 9, 359-378 (2018), arXiv:1704.06660 [condmat.mes-hall].

[11] D. T. Son and B. Z. Spivak, "Chiral Anomaly and Classical Negative Magnetoresistance of Weyl Metals," Phys. Rev. B88, 104412 (2013), arXiv:1206.1627 [condmat.mes-hall].

[12] Qiang Li, Dmitri E. Kharzeev, Cheng Zhang, Yuan Huang, I. Pletikosic, A. V. Fedorov, R. D. Zhong, J. A. Schneeloch, G. D. Gu, and T. Valla, "Observation of the chiral magnetic effect in ZrTe5," Nature Phys. 12, 550-554 (2016), arXiv:1412.6543 [cond-mat.str-el].

[13] Xiaochun Huang, Lingxiao Zhao, Yujia Long, Peipei Wang, Dong Chen, Zhanhai Yang, Hui Liang, Mianqi Xue, Hongming Weng, Zhong Fang, Xi Dai, and Genfu Chen, "Observation of the chiral-anomaly-induced negative magnetoresistance in $3 \mathrm{~d}$ weyl semimetal taas," Phys. Rev. X 5, 031023 (2015).

[14] Frank Arnold et al., "Negative magnetoresistance without well-defined chirality in the Weyl semimetal TaP," Nature Commun. 7, 1615 (2016), arXiv:1506.06577 [cond-mat.mtrl-sci].

[15] Dmitri E. Kharzeev, Larry D. McLerran, and Harmen J. Warringa, "The Effects of topological charge change in heavy ion collisions: 'Event by event $\mathrm{P}$ and CP violation'," Nucl. Phys. A803, 227-253 (2008), arXiv:0711.0950 [hep-ph].

[16] Kenji Fukushima, Dmitri E. Kharzeev, and Harmen J. Warringa, "The Chiral Magnetic Effect," Phys. Rev. D78, 074033 (2008), arXiv:0808.3382 [hep-ph].

[17] B. I. Abelev et al. (STAR), "Azimuthal Charged-Particle Correlations and Possible Local Strong Parity Violation," 
Phys. Rev. Lett. 103, 251601 (2009), arXiv:0909.1739 [nucl-ex].

[18] B. I. Abelev et al. (STAR), "Observation of chargedependent azimuthal correlations and possible local strong parity violation in heavy ion collisions," Phys. Rev. C81, 054908 (2010), arXiv:0909.1717 [nucl-ex].

[19] L. Adamczyk et al. (STAR), "Beam-energy dependence of charge separation along the magnetic field in $\mathrm{Au}+\mathrm{Au}$ collisions at RHIC," Phys. Rev. Lett. 113, 052302 (2014), arXiv:1404.1433 [nucl-ex].

[20] Betty Abelev et al. (ALICE), "Charge separation relative to the reaction plane in $\mathrm{Pb}-\mathrm{Pb}$ collisions at $\sqrt{s_{N N}}=2.76 \mathrm{TeV}$," Phys. Rev. Lett. 110, 012301 (2013), arXiv:1207.0900 [nucl-ex].

[21] Shreyasi Acharya et al. (ALICE), "Constraining the magnitude of the Chiral Magnetic Effect with Event Shape Engineering in $\mathrm{Pb}-\mathrm{Pb}$ collisions at $\sqrt{s_{\mathrm{NN}}}=2.76 \mathrm{TeV}$," Phys. Lett. B777, 151-162 (2018), arXiv:1709.04723 [nucl-ex].

[22] Vardan Khachatryan et al. (CMS), "Observation of charge-dependent azimuthal correlations in $p-\mathrm{Pb}$ collisions and its implication for the search for the chiral magnetic effect," Phys. Rev. Lett. 118, 122301 (2017), arXiv:1610.00263 [nucl-ex].

[23] Albert M Sirunyan et al. (CMS), "Constraints on the chiral magnetic effect using charge-dependent azimuthal correlations in $p \mathrm{~Pb}$ and $\mathrm{PbPb}$ collisions at the CERN Large Hadron Collider," Phys. Rev. C97, 044912 (2018), arXiv:1708.01602 [nucl-ex].

[24] Adam Bzdak, Volker Koch, and Jinfeng Liao, "ChargeDependent Correlations in Relativistic Heavy Ion Collisions and the Chiral Magnetic Effect," Lect. Notes Phys. 871, 503-536 (2013), arXiv:1207.7327 [nucl-th].

[25] Jie Zhao and Fuqiang Wang, "Experimental searches for the chiral magnetic effect in heavy-ion collisions," Prog. Part. Nucl. Phys. 107, 200-236 (2019), arXiv:1906.11413 [nucl-ex].

[26] Sergei A. Voloshin, "Testing the Chiral Magnetic Effect with Central U+U collisions," Phys. Rev. Lett. 105, 172301 (2010), arXiv:1006.1020 [nucl-th].

[27] Volker Koch, Soeren Schlichting, Vladimir Skokov, Paul Sorensen, Jim Thomas, Sergei Voloshin, Gang Wang, and Ho-Ung Yee, "Status of the chiral magnetic effect and collisions of isobars," Chin. Phys. C41, 072001 (2017), arXiv:1608.00982 [nucl-th].

[28] D. E. Kharzeev and J. Liao, "Isobar Collisions at RHIC to Test Local Parity Violation in Strong Interactions," Nucl. Phys. News 29, 26-31 (2019).

[29] John Bloczynski, Xu-Guang Huang, Xilin Zhang, and Jinfeng Liao, "Azimuthally fluctuating magnetic field and its impacts on observables in heavy-ion collisions," Phys. Lett. B718, 1529-1535 (2013), arXiv:1209.6594 [nucl-th].

[30] Chun Shen, Zhi Qiu, Huichao Song, Jonah Bernhard, Steffen Bass, and Ulrich Heinz, "The iEBE-VISHNU code package for relativistic heavy-ion collisions," Comput. Phys. Commun. 199, 61-85 (2016), arXiv:1409.8164 [nucl-th].

[31] Charles Gale, Sangyong Jeon, and Bjoern Schenke, "Hydrodynamic Modeling of Heavy-Ion Collisions," Int. J. Mod. Phys. A28, 1340011 (2013), arXiv:1301.5893 [nuclth].

[32] Wei-Tian Deng, Xu-Guang Huang, Guo-Liang Ma, and Gang Wang, "Test the chiral magnetic effect with isobaric collisions," Phys. Rev. C94, 041901 (2016), arXiv:1607.04697 [nucl-th].

[33] Yifeng Sun and Che Ming Ko, "Chiral kinetic approach to the chiral magnetic effect in isobaric collisions," Phys. Rev. C98, 014911 (2018), arXiv:1803.06043 [nucl-th].

[34] Xin-Li Zhao, Guo-Liang Ma, and Yu-Gang Ma, "Impact of magnetic-field fluctuations on measurements of the chiral magnetic effect in collisions of isobaric nuclei," Phys. Rev. C99, 034903 (2019), arXiv:1901.04151 [hep-ph].

[35] Dam T. Son and Piotr Surowka, "Hydrodynamics with Triangle Anomalies," Phys. Rev. Lett. 103, 191601 (2009), arXiv:0906.5044 [hep-th].

[36] Shuzhe Shi, Yin Jiang, Elias Lilleskov, and Jinfeng Liao, "Anomalous Chiral Transport in Heavy Ion Collisions from Anomalous-Viscous Fluid Dynamics," Annals Phys. 394, 50-72 (2018), arXiv:1711.02496 [nucl-th].

[37] Yin Jiang, Shuzhe Shi, Yi Yin, and Jinfeng Liao, "Quantifying the chiral magnetic effect from anomalousviscous fluid dynamics," Chin. Phys. C42, 011001 (2018), arXiv:1611.04586 [nucl-th].

[38] Hao-Jie Xu, Xiaobao Wang, Hanlin Li, Jie Zhao, ZiWei Lin, Caiwan Shen, and Fuqiang Wang, "Importance of isobar density distributions on the chiral magnetic effect search," Phys. Rev. Lett. 121, 022301 (2018), arXiv:1710.03086 [nucl-th].

[39] Shuzhe Shi, Hui Zhang, Defu Hou, and Jinfeng Liao, "Chiral Magnetic Effect in Isobaric Collisions from Anomalous-Viscous Fluid Dynamics (AVFD)," Proceedings, 27th International Conference on Ultrarelativistic Nucleus-Nucleus Collisions (Quark Matter 2018): Venice, Italy, May 14-19, 2018, Nucl. Phys. A982, 539542 (2019), arXiv:1807.05604 [hep-ph].

[40] Jan Hammelmann, Alba Soto-Ontoso, Massimiliano Alvioli, Hannah Elfner, and Mark Strikman, "Influence of the neutron-skin effect on nuclear isobar collisions at RHIC," arXiv (2019), arXiv:1908.10231 [nucl-th].

[41] L. McLerran and V. Skokov, "Comments About the Electromagnetic Field in Heavy-Ion Collisions," Nucl. Phys. A929, 184-190 (2014), arXiv:1305.0774 [hep-ph].

[42] Umut Gursoy, Dmitri Kharzeev, and Krishna Rajagopal, "Magnetohydrodynamics, charged currents and directed flow in heavy ion collisions," Phys. Rev. C89, 054905 (2014), arXiv:1401.3805 [hep-ph].

[43] Kirill Tuchin, "Initial value problem for magnetic fields in heavy ion collisions," Phys. Rev. C93, 014905 (2016), arXiv:1508.06925 [hep-ph].

[44] Gabriele Inghirami, Luca Del Zanna, Andrea Beraudo, Mohsen Haddadi Moghaddam, Francesco Becattini, and Marcus Bleicher, "Numerical magneto-hydrodynamics for relativistic nuclear collisions," Eur. Phys. J. C76, 659 (2016), arXiv:1609.03042 [hep-ph].

[45] Umut Gürsoy, Dmitri Kharzeev, Eric Marcus, Krishna Rajagopal, and Chun Shen, "Charge-dependent Flow Induced by Magnetic and Electric Fields in Heavy Ion Collisions," Phys. Rev. C98, 055201 (2018), arXiv:1806.05288 [hep-ph].

[46] Victor Roy, Shi Pu, Luciano Rezzolla, and Dirk H. Rischke, "Effect of intense magnetic fields on reducedMHD evolution in $\sqrt{s_{\mathrm{NN}}}=200 \mathrm{GeV} \mathrm{Au+Au} \mathrm{collisions,"}$ Phys. Rev. C96, 054909 (2017), arXiv:1706.05326 [nuclth].

[47] Shi Pu, Victor Roy, Luciano Rezzolla, and Dirk H. Rischke, "Bjorken flow in one-dimensional relativistic magnetohydrodynamics with magnetization," Phys. Rev. 
D93, 074022 (2016), arXiv:1602.04953 [nucl-th].

[48] Berndt Müller and Andreas Schäfer, "Chiral magnetic effect and an experimental bound on the late time magnetic field strength," Phys. Rev. D98, 071902 (2018), arXiv:1806.10907 [hep-ph].

[49] Yu Guo, Shuzhe Shi, Shengqin Feng, and Jinfeng Liao, "Magnetic Field Induced Polarization Difference between Hyperons and Anti-hyperons," Phys. Lett. B798, 134929 (2019), arXiv:1905.12613 [nucl-th].

[50] Xingyu Guo, Jinfeng Liao, and Enke Wang, "Magnetic Field in the Charged Subatomic Swirl," (2019), arXiv:1904.04704 [hep-ph].

[51] Sergei A. Voloshin, "Parity violation in hot QCD: How to detect it," Phys. Rev. C70, 057901 (2004), arXiv:hepph/0406311 [hep-ph].

[52] Adam Bzdak, Volker Koch, and Jinfeng Liao, "Remarks on possible local parity violation in heavy ion collisions," Phys. Rev. C81, 031901 (2010), arXiv:0912.5050 [nuclth].

[53] Soren Schlichting and Scott Pratt, "Charge conservation at energies available at the BNL Relativistic Heavy Ion Collider and contributions to local parity violation observables," Phys. Rev. C83, 014913 (2011), arXiv:1009.4283 [nucl-th].

[54] John Bloczynski, Xu-Guang Huang, Xilin Zhang, and Jinfeng Liao, "Charge-dependent azimuthal correlations from AuAu to UU collisions," Nucl. Phys. A939, 85-100 (2015), arXiv:1311.5451 [nucl-th].

[55] Fufang Wen, Jacob Bryon, Liwen Wen, and Gang Wang, "Event-shape-engineering study of charge separation in heavy-ion collisions," Chin. Phys. C42, 014001 (2018), arXiv:1608.03205 [nucl-th].

[56] Hao-jie Xu, Jie Zhao, Xiaobao Wang, Hanlin Li, ZiWei Lin, Caiwan Shen, and Fuqiang Wang, "Varying the chiral magnetic effect relative to flow in a sin- gle nucleus-nucleus collision," Chin. Phys. C42, 084103 (2018), arXiv:1710.07265 [nucl-th].

[57] Jie Zhao, Hanlin Li, and Fuqiang Wang, "Isolating the chiral magnetic effect from backgrounds by pair invariant mass," Eur. Phys. J. C79, 168 (2019), arXiv:1705.05410 [nucl-ex].

[58] Ling Huang, Mao-Wu Nie, and Guo-Liang Ma, "Sensitivity analysis of the chiral magnetic effect observables using a multiphase transport model," (2019), arXiv:1906.11631 [nucl-th].

[59] Subikash Choudhury, Gang Wang, Wanbing He, Yu Hu, and Huan Zhong Huang, "Background evaluations for the chiral magnetic effect with normalized correlators using a multiphase transport model," (2019), arXiv:1909.04083 [hep-ph].

[60] Sergei A. Voloshin, "Estimate of the signal from the chiral magnetic effect in heavy-ion collisions from measurements relative to the participant and spectator flow planes," Phys. Rev. C 98, 054911 (2018), arXiv:1805.05300 [nucl-ex].

[61] Niseem Magdy, Shuzhe Shi, Jinfeng Liao, N. Ajitanand, and Roy A. Lacey, "New correlator to detect and characterize the chiral magnetic effect," Phys. Rev. C97, 061901 (2018), arXiv:1710.01717 [physics.data-an].

[62] Niseem Magdy, Shuzhe Shi, Jinfeng Liao, Peifeng Liu, and Roy A. Lacey, "Examination of the observability of a chiral magnetically driven charge-separation difference in collisions of the ${ }_{44}^{96} \mathrm{Ru}+{ }_{44}^{96} \mathrm{Ru}$ and ${ }_{40}^{96} \mathrm{Zr}+{ }_{40}^{96} \mathrm{Zr}$ isobars at energies available at the BNL Relativistic Heavy Ion Collider," Phys. Rev. C98, 061902 (2018), arXiv:1803.02416 [nucl-ex].

[63] A.H. Tang, "Probe chiral magnetic effect with signed balance function," Chin. Phys. C 44, 054101 (2020), arXiv:1903.04622 [nucl-ex]. 